\title{
Research Paper: \\ A Cross-sectional Study on the Ability to Remember Mania Symptoms in Patients With Type I Bipolar Disorder 60 Months After Hospital Discharge
}

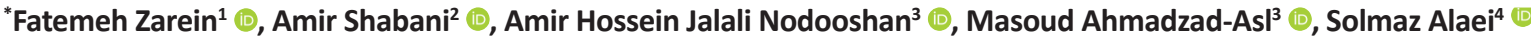

1. Resident of Psychiatry, Department of Psychiatry, School of Medicine, Iran University of Medical Sciences, Tehran, Iran.

2. Mental Health Research Center, Mood Disorders Research Group, Iran University of Medical Sciences, Tehran, Iran.

3. Mental Health Research Center, Community Mental Health Research Group, Iran University of Medical Sciences, Tehran, Iran.

4. Fellowship in Psychotherapy, Department of Psychiatry, School of Medicine, Tehran University of Medical Sciences, Tehran, Iran.

\begin{tabular}{|c|c|}
\hline $\begin{array}{l}\text { Use vourdevice to scan } \\
\text { and read the article online }\end{array}$ & Ceftertion Zarein F, Shabani A, Jalali A, Ahmadzad-Asl M, Alaei S. A Cross-sectional Study on the Ability to Remember Mania \\
\hline 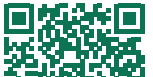 & $\begin{array}{l}\text { Symptoms in Patients With Type I Bipolar Disorder } 60 \text { Months After Hospital Discharge .Iranian Journal of Psychiatry and Clini- } \\
\text { cal Psychology. 2020; 25(4):428-439. http://dx.doi.org/10.32598/ijpcp.25.4.6 }\end{array}$ \\
\hline 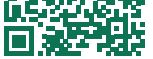 & doil'http://dx.doi.org/10.32598/ijpcp.25.4.6 \\
\hline
\end{tabular}

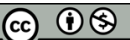

Received: 22 Apr 2019

Accepted: 31 Aug 2019

Available Online: 01 Jan 2020

Key words:

Manic episode, Bipolar disorder, Psychiatrists, Irritability

\section{A B S T R A C T}

Objectives Identifying the acute symptoms of manic episode remembered by the patients can help psychiatrists improve their ability to manage bipolar disorder. Given the importance of remembering symptoms, the aim of this study was to evaluate the ability of patients with type I bipolar disorder during the euthymic period to remember their past mania symptoms after hospital discharge.

Methods Participants were 59 patients with type I bipolar disorder admitted to Hazrat-e-Rasool-e-Akram Hospital and Iran Psychiatry Hospital in 2012. They measured by using Structured Clinical Interview for DSM-IV axis I Disorders (SCID-I) and Young Mania Rating Scale (YMRS) and their mania symptoms were recorded. Their demographic information were extracted from their medical records. Sixth months after discharge, they were evaluated again by using the 17-item Hamilton Depression Rating Scale (HRSD-17), SCID-I, YMRS, and Mini-Mental State Examination (MMSE) through face-to-face interview. To assess the agreement on the symptoms during admission and 60 months after discharge, McNemar Test and Kappa coefficients were used.

Results most common mania symptoms remembered by the patients were decreased need for sleep (91.2\%), irritability (83.9\%), excessive involvement in activities with a high likelihood of painful consequences (81.3\%) and the least frequent remembered symptom was distractibility (17.6\%). The highest positive predictive values were related to the symptoms of irritability $(100 \%)$, talkativeness $(100 \%)$ and decreased need for sleep (96.3\%), while the highest negative predictive value was related to elevated $\operatorname{mood}(87.5 \%)$.

Conclusion management of patients with bipolar disorder, psychiatrists can trust the patients' ability to remember the three mania symptoms including irritability, decreased need for sleep and talkativeness. Regarding the symptom of elevated mood in bipolar patients, negative response of patients can be trusted.

\section{Extended Abstract}

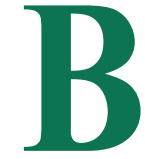

\section{Introduction}

ipolar disorder is a kind of chronic illness with recurrent mood episodes that can lead to severe impairments in the one's social and occupational performance [1]. In the present study, using the cases studied by Alaei et al. [5] and the symptoms remembered by patients in the euthymic phase after discharge from hospital, it is aimed to identify the most common symptoms remained

* Corresponding Author:

Fatemeh Zarein, PhD.

Address: Department of Psychiatry, School of Medicine, Iran University of Medical Sciences, Tehran, Iran.

Tel: +98 (912) 3122492

E-mail: dr.zarein@gmail.com 
in the memory of patients as far as possible in order to help psychiatrists to diagnose a previous episode of this illness and, therefore, provide better and faster patient care. Therefore, a comprehensive knowledge of bipolar disorder pattern over time, extracting the past maniac episodes from the patients' records for the preparation of life charts, selection of appropriate treatment (pharmacological/non-pharmacological), determining prognosis, planning for follow-up and maintaining treatment are important [3]. The purpose of this study is to investigate the most common symptoms that remembered by patients with bipolar disorder after the maniac phase, by examining the extent of remembering the different symptoms of mania (psychotic symptoms including hallucination and delusion)

\section{Method}

The study samples includes 59 patients with type I bipolar disorder who were admitted to Hazrat-e-Rasool-e-Akhram Hospital and Iran Psychiatry Hospital in 2012. Their inclusion criteria were participation in the study conducted by Alaei et al. [5]. Structured Clinical Interview for DSM-IV axis I Disorders (SCID-I) and Young Mania Rating Scale (YMRS) were completed for these patients, and their mania symptoms were recorded. Demographic information of these patients was also extracted from their medical records. In the first step, $60 \pm 2$ months after discharge from hospital, the patients were invited by telephone. After excluding 37 cases (38.5\%), 59 samples $(61.5 \%)$ were interviewed by face-to-face method. After receiving written informed consent from them, the symptoms remembered by them were extracted and recorded using the SCID-I. The researchers also completed the Hamilton Depression Rating Scale (HRSD), YMRS, and Mini-Mental State Examination (MMSE) for the patients.

\section{Results}

The most common symptom of mania in patients that is remembered $60 \pm 2$ months after discharge was "decreased need for sleep". Also, there was a significant difference in the extent that they were able to remember the three symptoms of "decreased need for sleep", "excessive involvement in pleasurable activity" and "psychotic symptoms" between 1 and $60 \pm 2$ months after discharge. According to Table 1, the most common symptom of mania remembered $60 \pm 2$ months after discharge by the patients was "decreased need for sleep".

The higher positive predictive values were observed in irritability (100\%), talkativeness (100\%), and decreased need for sleep (96.29\%) symptoms, while the higher negative predictive values were related to elevated mood (72.97\%), excessive involvement in pleasurable activities $(76 \%)$, and distractibility $(73.80 \%)$ symptoms. Moreover, the higher sensitivity values were related to decreased need for sleep (91.22\%), irritability (83.92\%), and excessive involvement in pleasurable activities $(81.25 \%)$, and the higher specificity values were related to irritability $(100 \%)$, talkativeness (100\%), and psychotic symptoms $(96.42 \%)$.

\section{Discussion}

Table 1. Frequency of mania symptoms remembered by the patients during admission, and one and 60 months after discharge

\begin{tabular}{|c|c|c|c|c|c|}
\hline \multirow[b]{2}{*}{ Mania Symptoms } & \multicolumn{3}{|c|}{ No. (\%) } & \multirow[b]{2}{*}{ McNemar } & \multirow{2}{*}{$\begin{array}{c}\text { Kappa } \\
\text { Coefficient }\end{array}$} \\
\hline & During Admission & $\begin{array}{c}\text { One Month After } \\
\text { Discharge }\end{array}$ & $\begin{array}{l}\text { Sixty Months After } \\
\text { Discharge }\end{array}$ & & \\
\hline $\begin{array}{l}\text { Decreased need for } \\
\text { sleep* }\end{array}$ & 57 (96.9) & $40(70.2)$ & $52(91.2)$ & 0.000 & 0.369 \\
\hline Irritability & $56(94.9)$ & 44 (78.6) & 47 (83.9) & 0.55 & 0.358 \\
\hline $\begin{array}{l}\text { Excessive involvement in } \\
\text { pleasurable activities* }\end{array}$ & $32(54.2)$ & $15(46.9)$ & $26(81.3)$ & 0.00 & 0.338 \\
\hline Psychotic symptoms* & $31(52.5)$ & $13(41.9)$ & $21(67.7)$ & 0.021 & 0.390 \\
\hline High confidence & $42(71.2)$ & 26 (61.9) & $27(64.3)$ & 1.000 & 0.540 \\
\hline Talkativeness & $53(89.8)$ & 27 (50.9) & 27 (50.9) & 1.000 & 0.849 \\
\hline Elevated mood & $13(22)$ & $4(30.8)$ & $5(38.5)$ & 1.000 & 0.494 \\
\hline Distractibility & $15(25.4)$ & $6(40)$ & $3(20)$ & 0.250 & 0.545 \\
\hline Psychomotor agitation & 49 (83.1) & $30(61.2)$ & $25(51)$ & 0.227 & 0.549 \\
\hline
\end{tabular}

* Significantly different between one month and 60 months after discharge $(\mathrm{P}<0.05)$ 
Most of the symptoms remembered 60 months after discharge by the patients were "decreased need for sleep" and "irritability", which were also reported by Alaei et al. [5] as the most commonly observed symptoms in one month after discharge. The higher value of irritability can be due to its unpleasant effects on the patient and the people around $\mathrm{him} / \mathrm{her}$, which needs to be treated in admission form [5]. The significant difference in remembering the mania symptoms of decreased need for sleep, excessive involvement in pleasurable activities, and psychotic symptoms can be attributed to increased disease knowledge, being in the euthymic phase, and the withdrawal of patients with cognitive problems as well as the difference in the type of interview and interviewer. It seems that as more time passes since the onset of bipolar disorder, and due to frequent referral and communication of patients with the treatment system as well as possible education on the illness, patients get more information about their illness and its symptoms, and thus increase their insight and recall of past mania symptoms [4]. The frequency of hospitalization [2] can be another reason which was not studied in this study.

The three mania symptoms of "irritability", "decreased need for sleep", and "talkativeness" had the highest positive predictive values; this finding is consistent with the findings of the study conducted by Alaei et al. "Elevated mood" had the highest negative predictive value in our study and in Alaei et al.'s study. These results can make the therapist more confident that the patient has a history of manic episode and thus help identify the past maniac episode. Dejection of the presence of elevated mood by the patient also can indicate the therapist that there is no need to seek further to find the symptom in the patient's records.

\section{Conclusion}

It can be concluded that the mania symptoms of irritability, talkativeness, and decreased need for sleep are the most important symptoms with positive predictive values. The recall of mania symptoms is reliable even long after hospital discharge. In this regard, clinicians can be assured of their diagnosis of past maniac episodes and the course of bipolar disorder. In case of longitudinal research, therapists are recommended to keep in touch with their patients, and include outpatients in their study population.

\section{Ethical Considerations}

\section{Compliance with ethical guidelines}

This study was extracted from the thesis of Fatemeh Zarein approved by the Research Ethics Committee of Iran University of Medical Sciences (Code: IR.IUMS.FMD.
REC.1396.9411286009). All ethical principles were considered in this study. All participants were informed of the study process, and then a written informed consent was obtained from them. They were free to leave the study at any time, and were assured of the confidentiality of their information.

\section{Funding}

This study received no fanatical support from any organization.

\section{Authors contributions}

All authors had contribution in preparing this article.

\section{Conflicts of interest}

The authors declare no conflict of interest. 


\title{
بررسى مقطعى توانايى يادآورى علائم مانيا در مبتلايان به اختلال دوقطبى نوع (، +7 ماه يس از ترخيص از بيمارستان
}

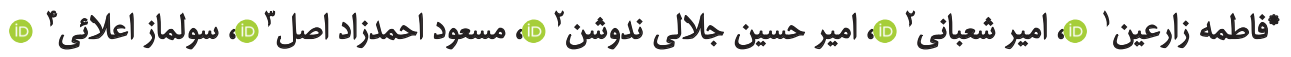

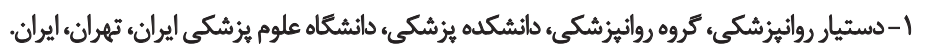

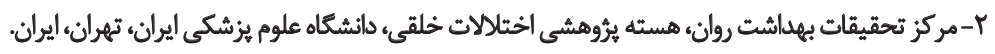

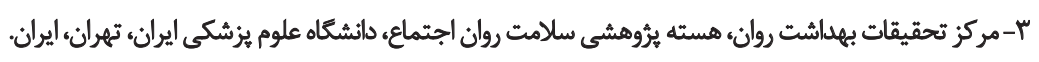

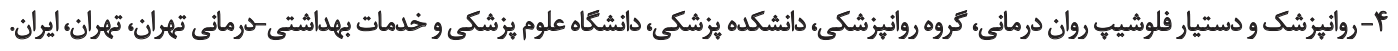

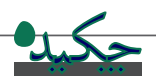

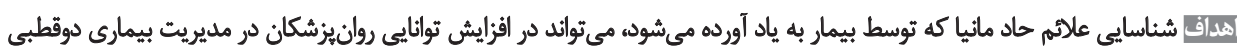

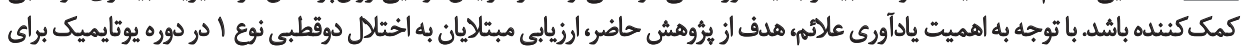

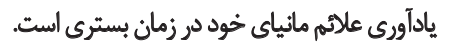

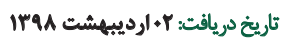

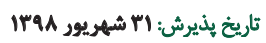
تاريخ انتشار: IIدى

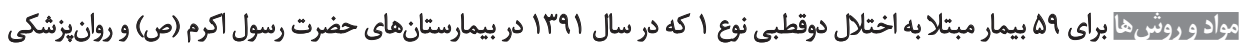

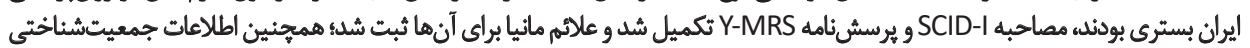

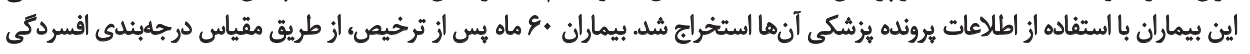

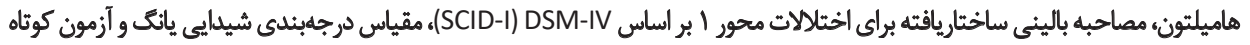

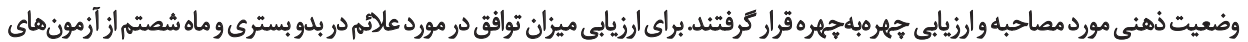

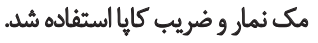

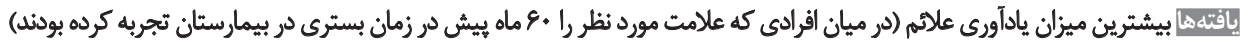

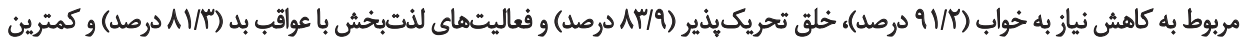

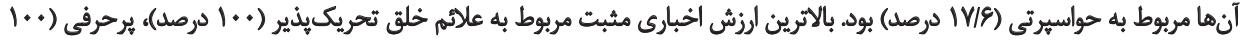

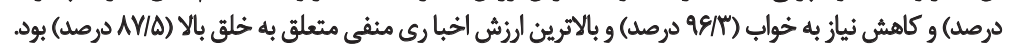

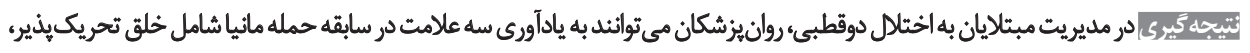

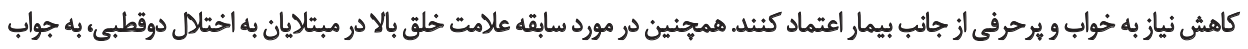
منفى بيمار ان ميى توان اعتماد كرد.

كليدوارهها:

اييزود مائيا، اختّلال

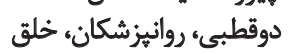
تحريك بذير
در تحقيق حاضر، با استفاده از نمونه مورد مطالعه علايى

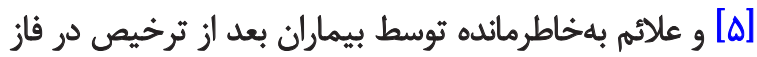

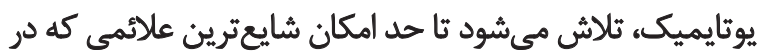

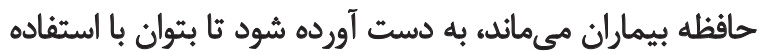

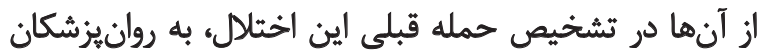

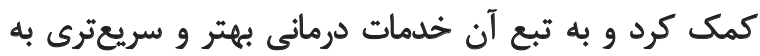

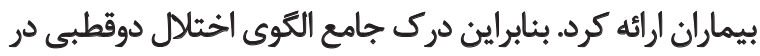

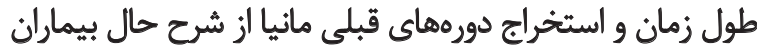

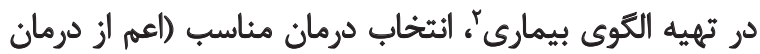

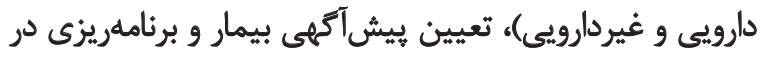

2. Life chart datêم

اختلال دوقطبى، نوعى اختلال خلقى مزمن و عودكنينده است

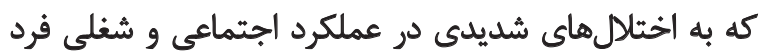

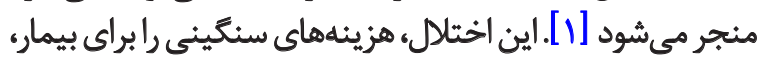

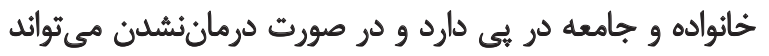

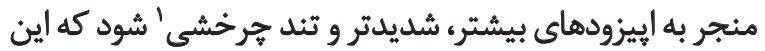

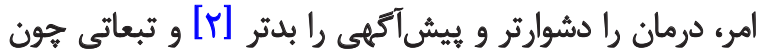

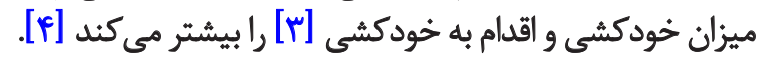

1. نوعى از بيمارى دوقطبى است كه در آنه بيمار حداقل جهار اييزود را در

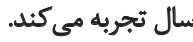

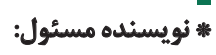
ناطمه زارينين

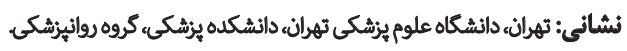
ت تلفئ: إيست الكترونيكى: dr.zarein@gmail.com 
شايعترين علامت در كل تحريكيديرى بود (در مردان شايعترين

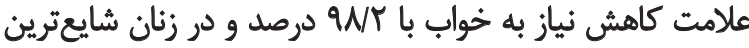

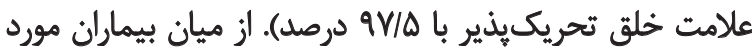

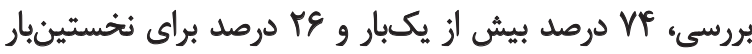

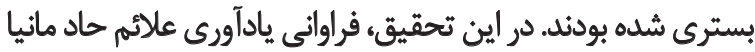

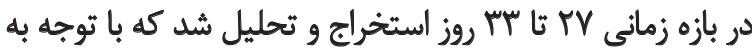

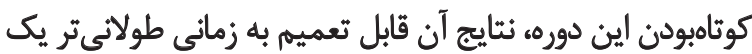
ماه نيست و در عمل به بازه زمانى طولانى تائرى نياز است.

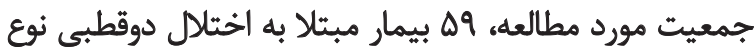

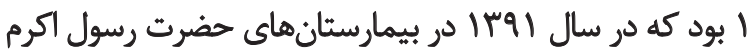

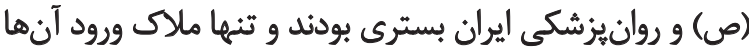

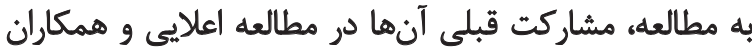

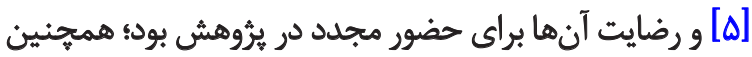

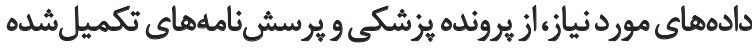
آنها در زمان بسترى استخراج شد.

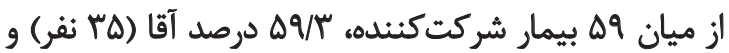

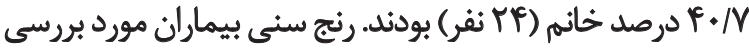

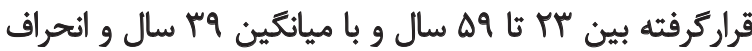

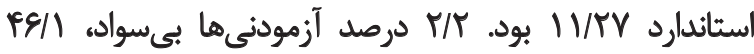

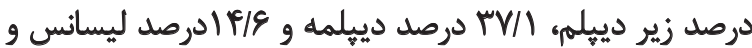

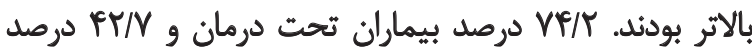
بيماران نيز در اين مدت سابقه بسترى مجدد داشتئد.

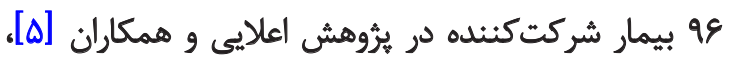

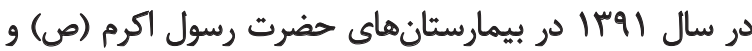

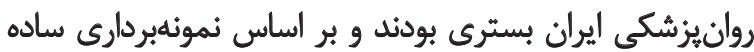

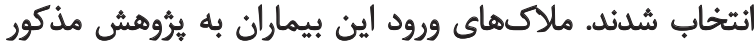

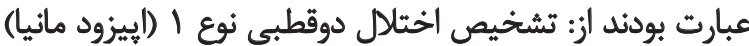

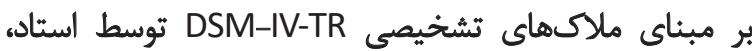

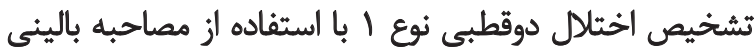

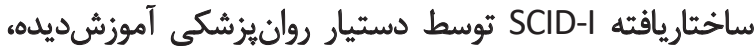

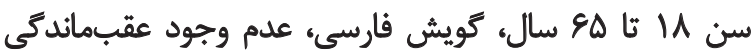

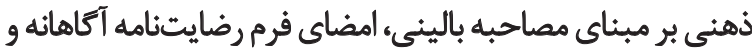
داشتن يك خط تلفن ثابت.

براي بيماران مذكور، مصاحبه ائيا

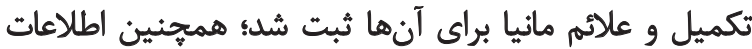

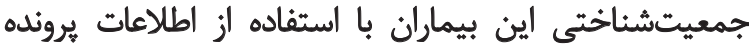

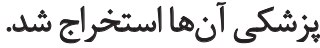

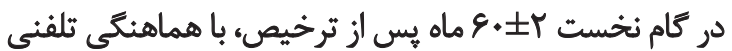

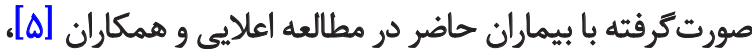

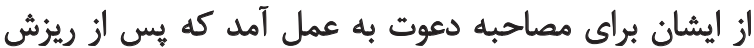

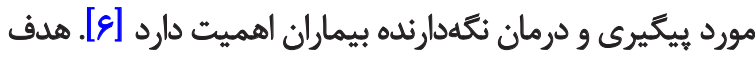

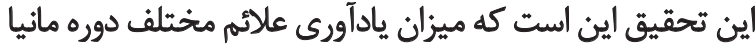

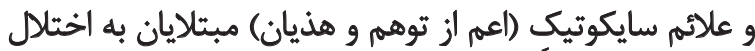

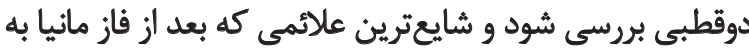
ياد مبتلايان به اختلال دوقطبى مى ماند، به دست آيدي

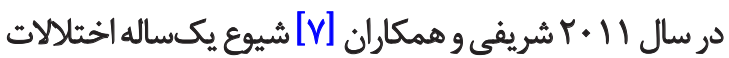

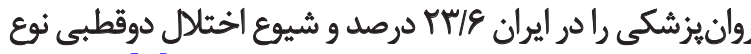

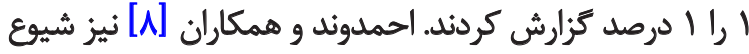

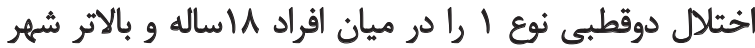

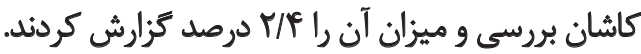

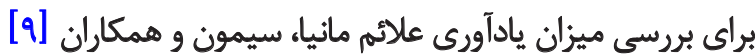

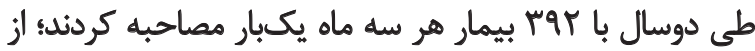

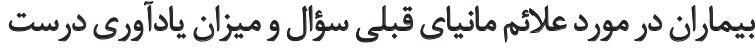

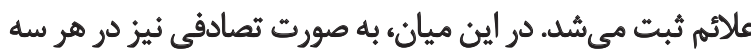

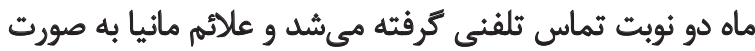

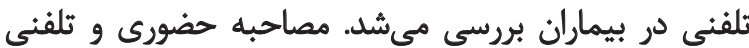

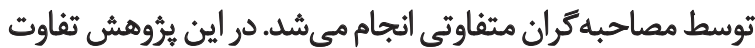
معنى دارى بين بررسى حضورى و تلفئنى علائم مانيا مشاهديد

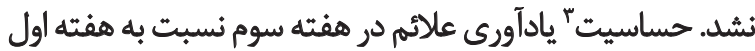

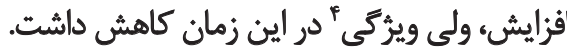

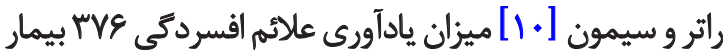

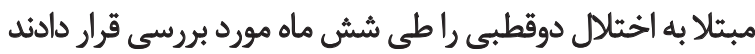

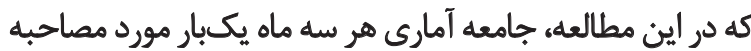

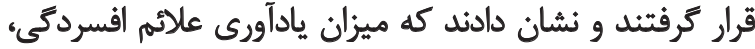

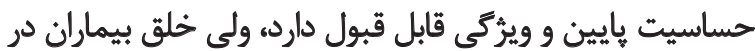

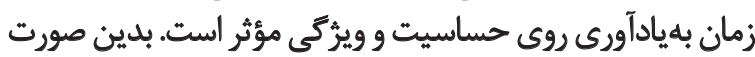

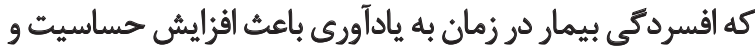

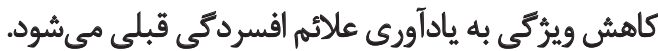

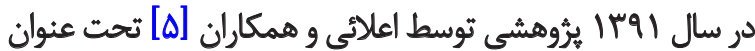

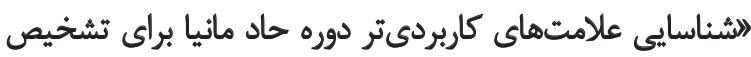

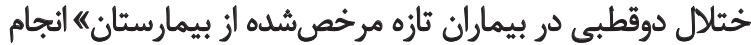

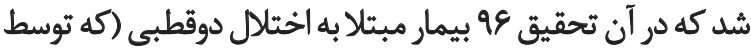

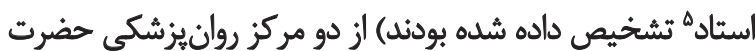

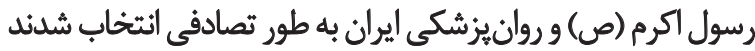

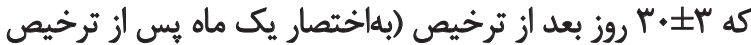

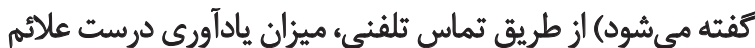

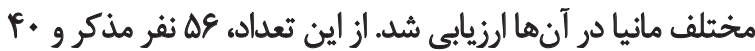

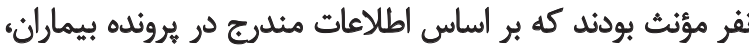

3. Sensitivity

4. Specificity

ه. منظور از استاد، عضو تقاموقت هيئت علمى دانشكاه علوميرشكى إنى

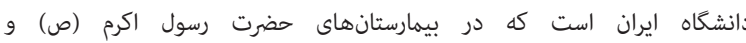
روانيزشكى ايران نيز فعاليت درمانى مى كنند. 


\section{مقياس درجهبندى افسردكى هاميلتون"}

يكى از رايجترين ابزارها براي تعيين شدت افسردئى الفي، مقياس

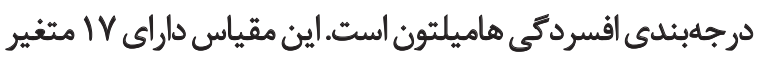

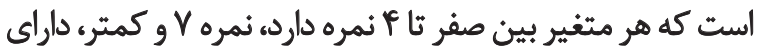

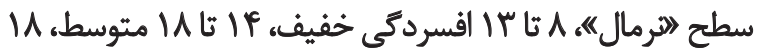

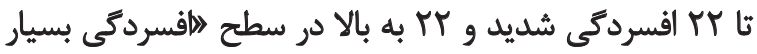

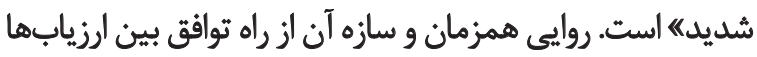

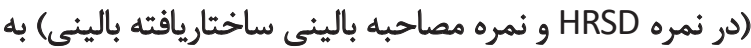

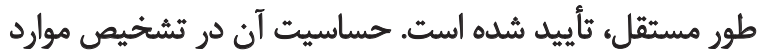

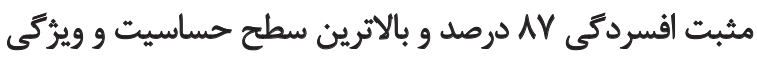

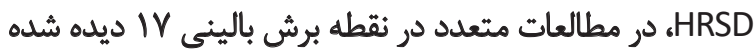

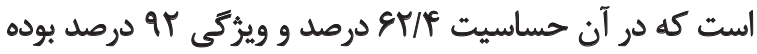

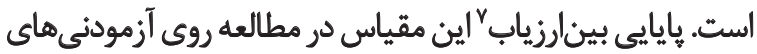

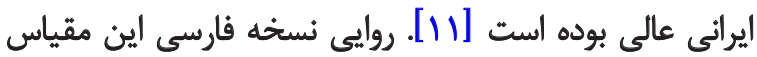

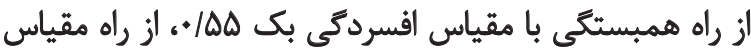

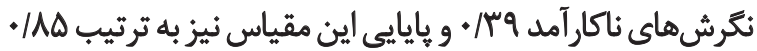

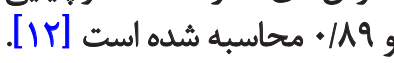

\section{مقياس درجدبندى شيدايى يانك^}

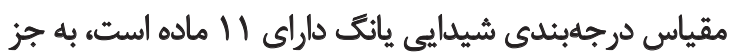

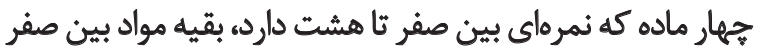

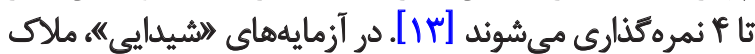

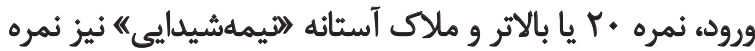

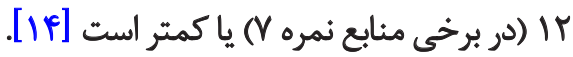

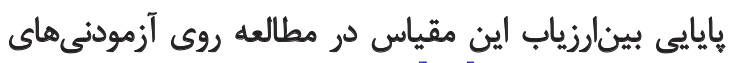

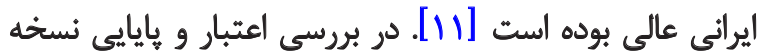

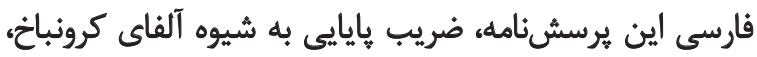

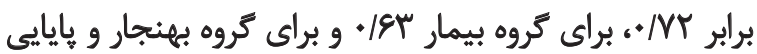

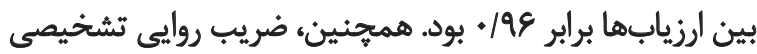

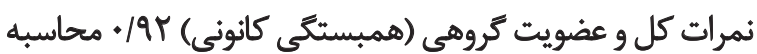

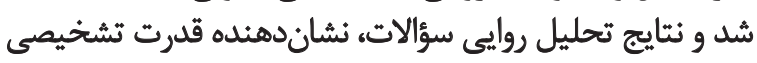

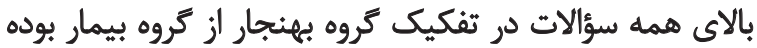

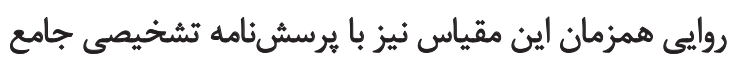

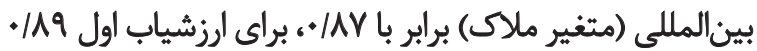

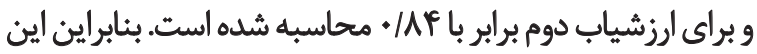

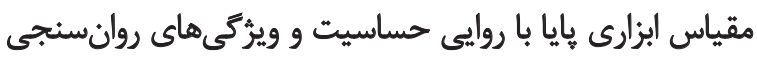
قابل قبول است كه قابليت استفاده در كارهاي باليت والينى و يثروهشى رونى

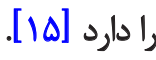

6. Hamilton Rating Scale for Depression (HRSD)

7. Inter-rater reliability

8. Young Mania Rating Scale (Y-MRS)

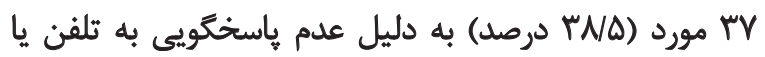

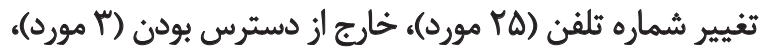

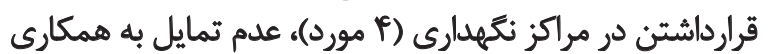

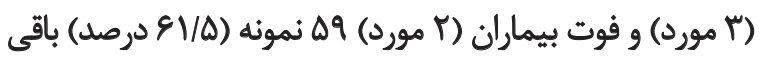
ماندند كه براي انجام مصاحبه حضورى مراجي (اجعه كردند.

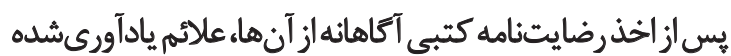

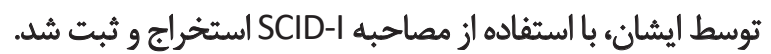

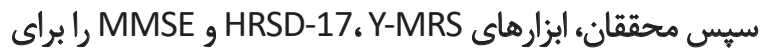

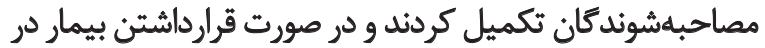

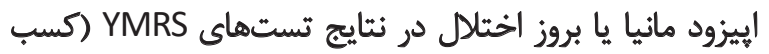

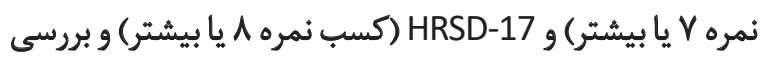

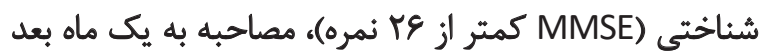

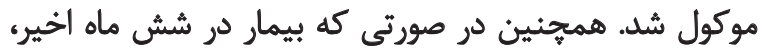

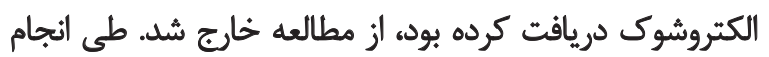

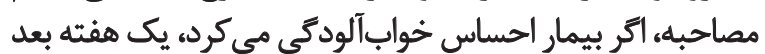
مورد مصاحبه قرار ميك يرفت.

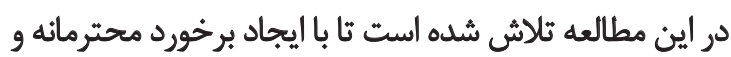

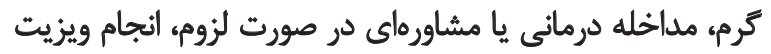

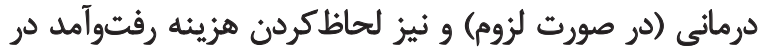

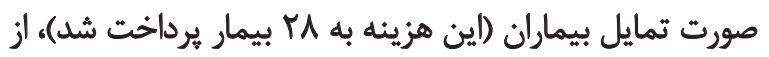
ريزش نمونهها جلوكيرى شود و همكارى بيمار ان افزايش يائه يابد.

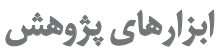

براى جمع آورى دادهها، علاوه بر يرسشنامه محققساخته كه

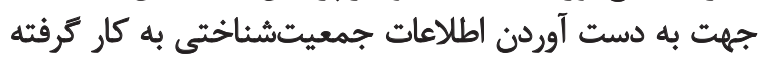
شد، از برسشنامهها و مقياس هاى ديكّرى نيز استفاده شد. برسش نامهويرُكى هاى جمعيتشناختى يرسش تامهاى شامل اطلاعات شخصى از جمله جنس، سن، سطح تحصيلات و وضعيت تأهل است

مصاحبه بالينى ساختاريافته براى اختلالات محور أبر (SCID-I) DSM-IV اساسباس باليني

SCID-I آن مستلزم قضاوت بالينى مصاحبه

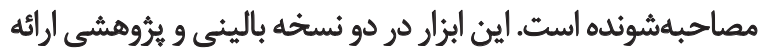

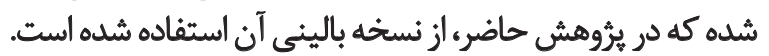

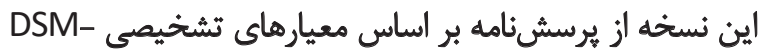

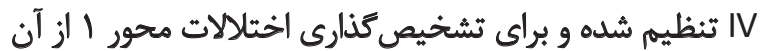

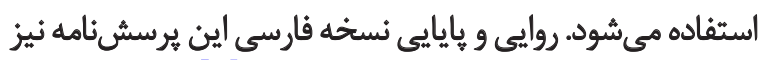

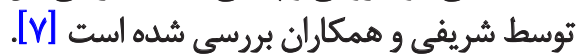


MMSE و و مصاحبه SCID و انجام شد. مصاحبهها به صورت انفرادى و با توجه به آمادكى بيماران تكميل شد و ويس إز إنكميل

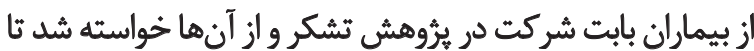
در نوبتهاى بعدى نيز همكارى كنيند.

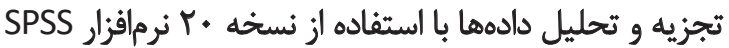

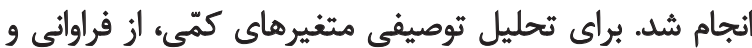

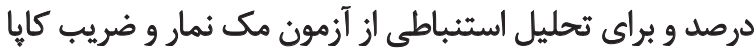

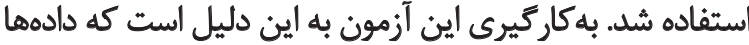

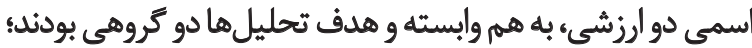

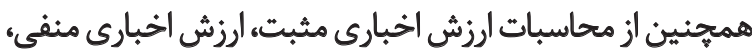
حساسيت و ويثزى نيز استفاده شد. اكر مقدار ضريب كايا صفر تا / / • باشد، بين مشاهده و

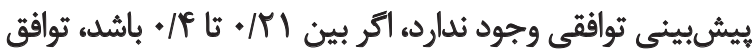

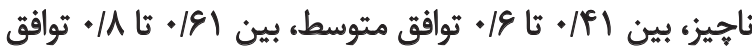

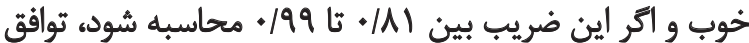

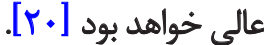

يأثتلها

جامعه آمارى اين تحقيق شامل ه9 بيمار است كه اطلاعات جمعيتشناختى آنها مطابق جدول شماره إمل است.

با توجه به جدول شماره Y و تصوير شماره (، بيشترين علامت

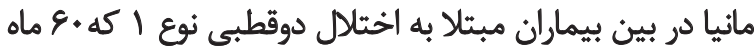

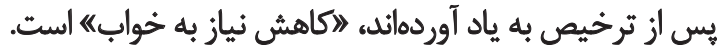
جدول شماره Y فراوانى علائم مانيا در بسترى مبدأ و ميزان

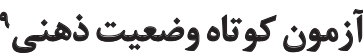

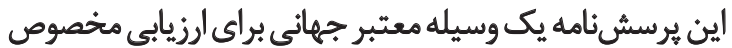

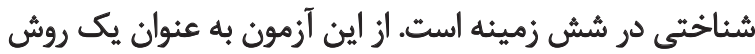

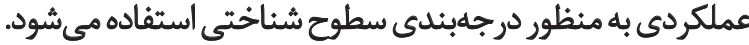

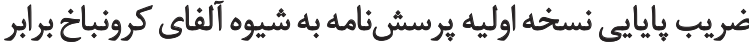

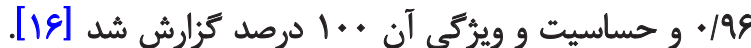

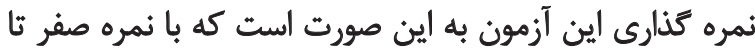

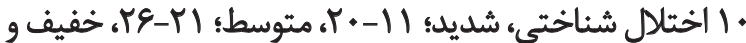

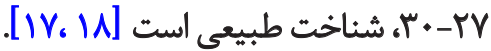

روايي افتراقى نسخه فارسى اين آزمون توسط سيديان و إنائ

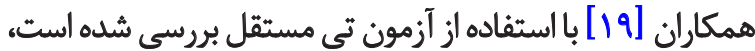

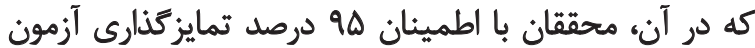

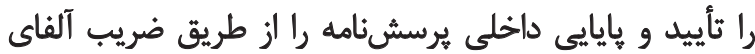

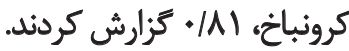

$$
\text { روش اجرأى يُوهش }
$$

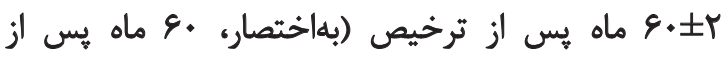

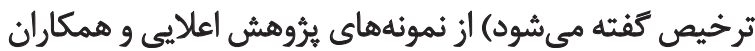

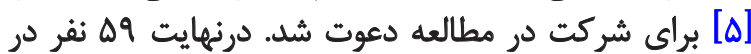

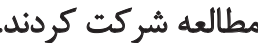
طى مصاحبهاي حضورى، اخذ رضايتنامه كتبى آكاهائه، ثبت

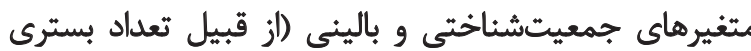

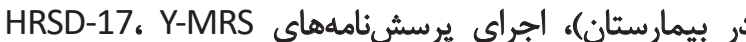

9. Mini-Mental State Examination (MMSE)

جدول ا. اطلاعات جمعيتشئاختى بيماران حاضر در برّوهش

\begin{tabular}{|c|c|c|c|c|}
\hline$(Y \circ / 7) ; Y Y)$ & 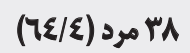 & 800 & & \\
\hline - & $r(1 .+)$ & $r$ & بيسواد & \multirow{4}{*}{ تحصيلات } \\
\hline$V(T \Delta)$ & $P(N \Delta)$ & $M$ & زير ديبلهم & \\
\hline . & . & - & دييلمي & \\
\hline$\| t(f f / A)$ & $1 \%(\Delta \Delta / T)$ & 99 & دائشكاهي & \\
\hline $\mid f(r / / A)$ & $r \cdot(E N Y)$ & pro & dب & \multirow[b]{2}{*}{ ثحت درمان } \\
\hline$\varepsilon(p+)$ & $9(8)$ & 10 & خير & \\
\hline$q(m / M)$ & $M(E \& M)$ & $M$ & بله & \multirow[b]{2}{*}{ بسترى مجلد } \\
\hline $11(m / r)$ & $r)(E \Delta / Y)$ & m & خير & \\
\hline$m q \pm 1 / / 1$ & $m q \pm 1 / / 8$ & $m+1 \| p$ & \multicolumn{2}{|c|}{ ميانكين سني } \\
\hline
\end{tabular}

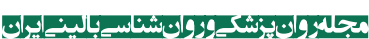


جدول ب. فراوائى علائم مانيا در بسترى مبدأ و ميزان يادآورى درست اين علائم يّ از يك ماه و .ع ماه

\begin{tabular}{|c|c|c|c|c|c|}
\hline \multirow{2}{*}{ ضريب كاها } & \multirow{2}{*}{ مكى نمار } & \multicolumn{3}{|c|}{ فراواثى (درصد) } & \multirow{2}{*}{ علانم مائيا } \\
\hline & & •7 ماه يس از ترخيص & يك ماه يس از ترخيص & فراوانى در بسترى مبدأ & \\
\hline - Req & $+\cdots$ & $\operatorname{Ar}(91 / T)$ & $F \cdot(V \cdot /)$ & $\Delta V(9 \& / 9)$ & كاهش نياز به خواب" \\
\hline.$/ T M A$ & $.10 \Delta$ & $\operatorname{Pr}(A r / q)$ & PF (VNE) & $\Delta \rho(94 / 9)$ & خلق تحريكيذير \\
\hline ( & $+1++1$ & $r \&(\Lambda \backslash / \pi)$ & $10(98 / 9)$ & $M(\Delta F / Y)$ & فعاليتهاي للتثبخش. \\
\hline.$/ 4 q$ & . H & $M(E N / V)$ & $1 r(p) / q)$ & $M(\Delta Y / \Delta)$ & علاثم سايكوتيك" \\
\hline . IAF. & $.1+1$ & $r V(E+/ T)$ & $r \&(81 / 9)$ & $M(M M / Y)$ & اعتمادبهنفس بالا \\
\hline - /Afeq & $.1 . .1$ & $\operatorname{rV}(\Delta \cdot / 9)$ & $\operatorname{rr}(\Delta . / 9)$ & $\Delta r(\Lambda q / \Lambda)$ & يرحرفى \\
\hline - /eque & .10 .1 & $\Delta(\Gamma N \Delta)$ & $f(r+/ \Lambda)$ & $M(M T)$ & خلق بالا \\
\hline . IAFD &.$/ T \Delta$ & $r(r \cdot)$ & $\varepsilon(f+)$ & $10(r \Delta / F)$ & حواسيبتى \\
\hline - $/ \Delta p q$ & . $M T Y$ & ra (A)) & $r \cdot(81 / T)$ & $p q(A r / N)$ & سراسيمكى \\
\hline
\end{tabular}

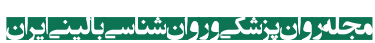

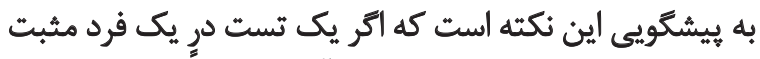
كزارش شود، جقدر احتمال دارد كه آن فرد واقعاً بيمار باشد. فئد

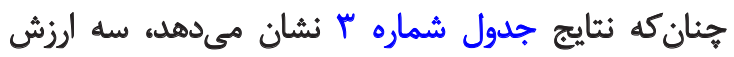

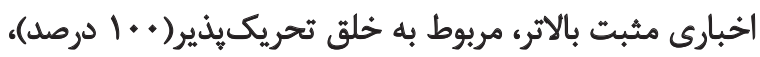

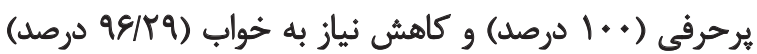

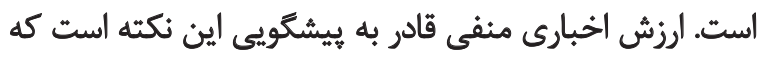

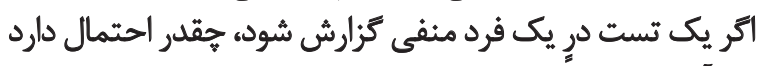

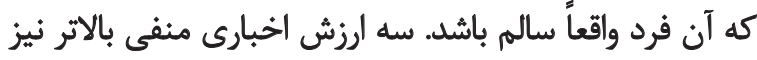

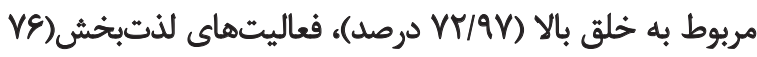

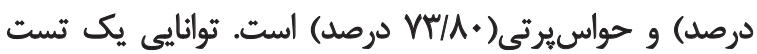

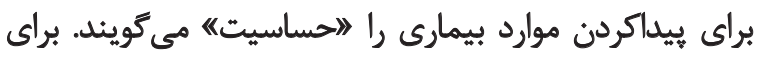

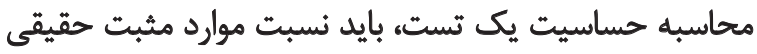

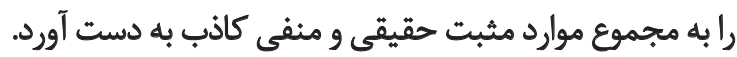
سه ارزش بالاتر حساسيت، مربوط به كاهش نياز به خوراب

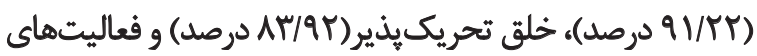

* داراى تفاوت معنيدار بين ماه يك و •ع (بار Pمتر هـ/.)

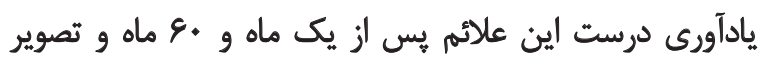

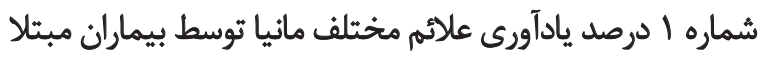

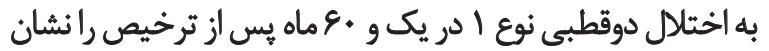

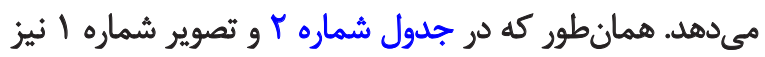

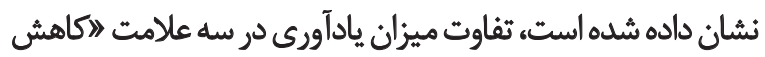

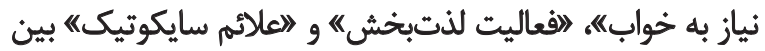
سنجش يك و •9 ماه هي از ترخيص، معنادار بود.

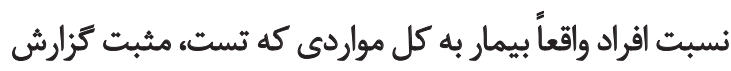

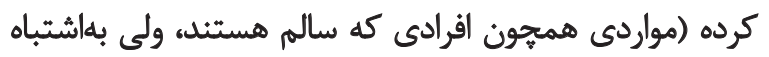

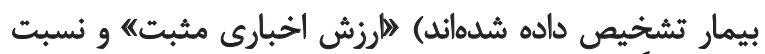

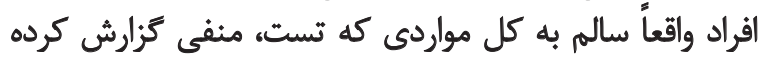

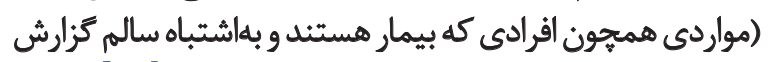

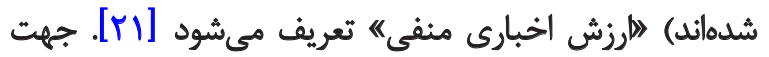

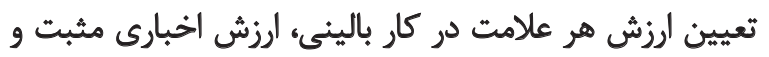

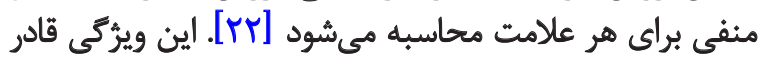

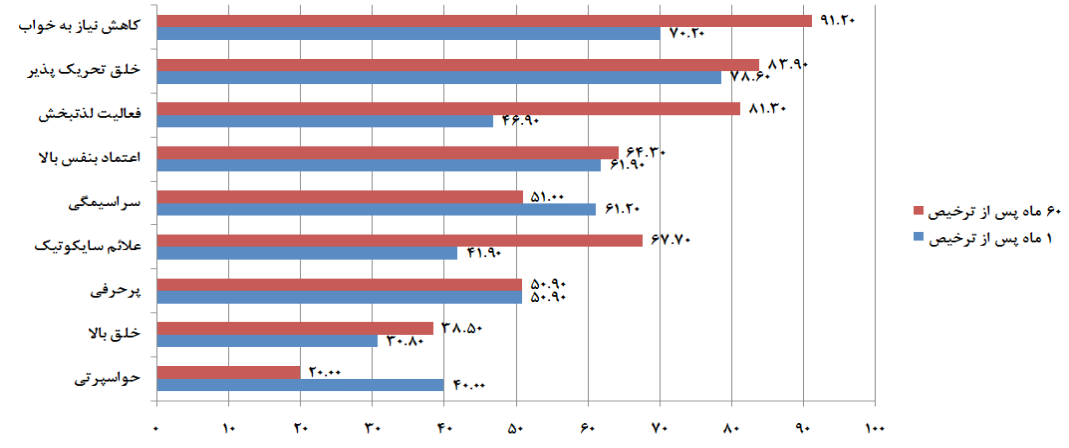

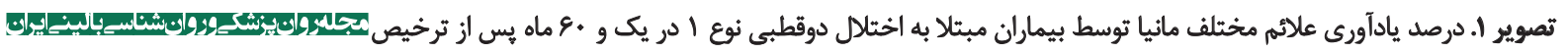


جدول ب.ا ارزش اخبارى مثبت و منفى، حساسيت و ويرّى هريك از علائم بهدستآمده مانيا در ماه .9

\begin{tabular}{|c|c|c|c|c|}
\hline \multirow{2}{*}{ ويزّكى } & \multirow{2}{*}{ حساسيت } & \multicolumn{2}{|c|}{ ارزش الخبارى } & \multirow{2}{*}{ Ne } \\
\hline & & منفى & مشبت & \\
\hline $1 .$. & AT/qY &.$/ T A$ & 1.0 & خلق تحريكائير \\
\hline$A Y / T \Delta$ & ex/TA & PNT & 9.1. & اعتمادبهنفس بالا \\
\hline .1. & $91 / \pi T$ & .1. & QS/Tq & كاهش نياز به خواب \\
\hline $1 \ldots$ & $0 . / 9 P^{\circ}$ & WVA & 1.0 & 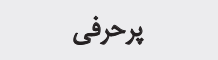 \\
\hline$V+/ 2 \Delta$ & $\mathrm{TI} / \mathrm{TH}$ & $n / \Lambda$. & IVIaY & حواسيرتي \\
\hline eqles & $0.1 \%$ & $19 / 20$ & A $/$ TA & سراسيمكى \\
\hline$V \cdot / m V$ & $A V / T \Delta$ & $\mathrm{V} \& 1 .$. & $V E / P V$ & فعاليتهاي للتبخش \\
\hline QE/TT & $S V / N F$ & $V \Upsilon / q V$ & 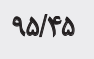 & علائم سايكوثيك \\
\hline$A V / \Delta$. & $P Q / F \Delta$ & $A V / \Delta$. & $P A / F A$ & خلق بالا \\
\hline
\end{tabular}

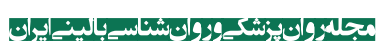

جدول f. همبستكى اسييرمن متغيرهاى مرتبط با يادآورى صحيح علاثم مانيا

\begin{tabular}{|c|c|c|c|c|c|}
\hline تحصيلات & جنسيت & بسترى مجدد & تحت درمانبودن & \multicolumn{2}{|c|}{ يادآورى صحيح در ماه } \\
\hline.$- / 1 P$ & $-+1+1$ &.$/ 1 \mathrm{~A}$ & -.1 .8 & Correlation Coefficient & \\
\hline.$/ 4 q$ &.$/ 91$ & .118 & .181 & p. value & يك ماه يس از ترخيص \\
\hline.$- / M r$ & .1 .0 &.$/ T A$ & -.1 .9 & Correlation Coefficient & \\
\hline$+1+1$ & .189 & $+1+\Delta$ &.$/ \Delta$ & p. value & 8. ماه هي \\
\hline
\end{tabular}

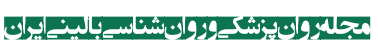

جدول هـ. ركرسيون خطى متغير سن با يادآورى صحيح علائم مانيا

\begin{tabular}{|c|c|c|}
\hline سن & \multicolumn{2}{|c|}{ يادآورى صحيح در ماه } \\
\hline .1 .6 & ضريب تعيين تعلديل شله & \\
\hline ITH $(\cdot / r \Delta H)$ & $F$ (p. value) & يك ماه هي أز ترخيص \\
\hline.$- / \cdot 10$ & ضريب تعيين تعديلشده & \\
\hline.$/ 11(\cdot / M m)$ & $F$ (p. value) & •و ماه يس از ترخيص \\
\hline
\end{tabular}

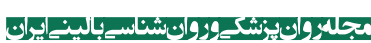

تحت درمان بودنه بسترى مجدد، جنسيت و تحصيلات الرتباطى با

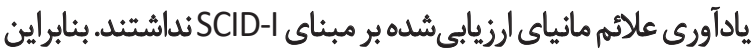

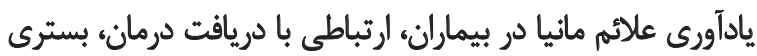

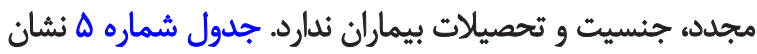

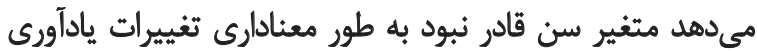

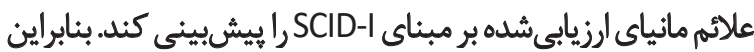

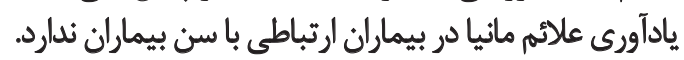

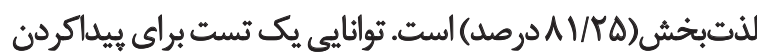

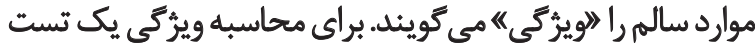

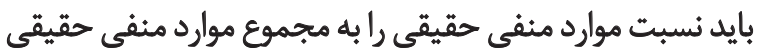

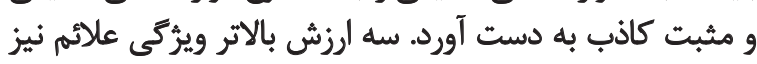

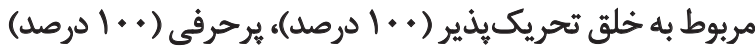

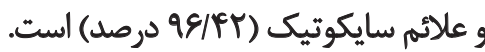

جنان كه جدول شماره f نشان ميدهد، هيجيك از متغيرهاى 
ور مورد يادآورى بيشتر دو علامت ديكر كاهش نياز به خواب

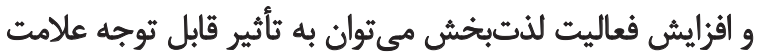

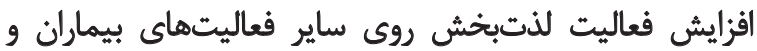

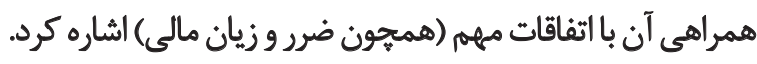
همجينين به همراه داشتن انكَ كمتر در يادآورى علامت كاهش

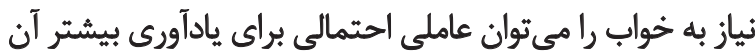

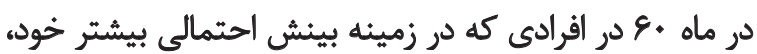

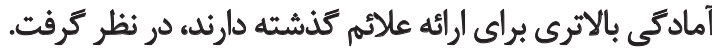
در رابطه با كمتر به ياد آورده شدن علامت سراسيمكى مي توان

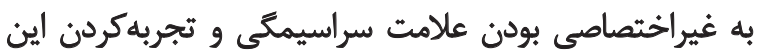

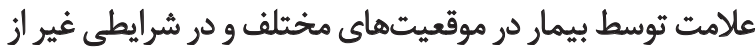
حمله مائيا اشاره كرد كه البته اين فرضيه نياز به بروسى بيته بيشتر

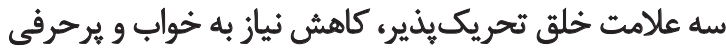

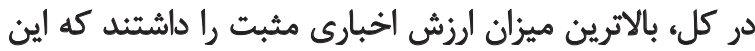

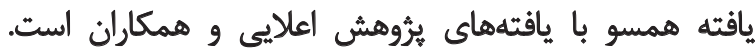

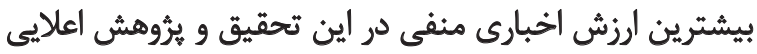

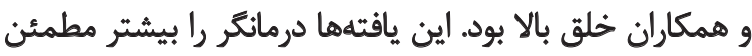

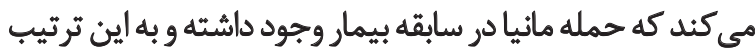

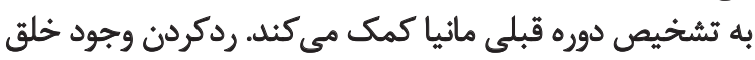

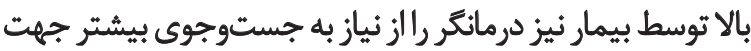

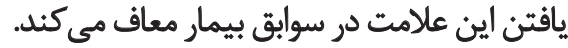

\section{تثيجليرى}

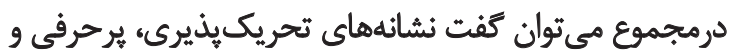

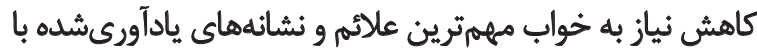

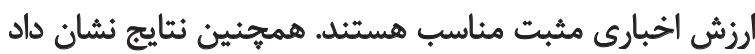

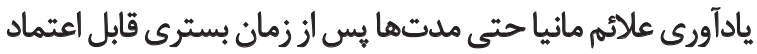

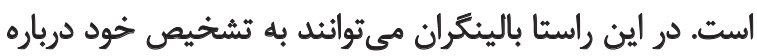

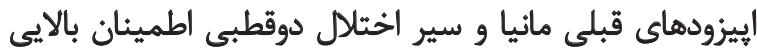

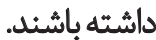

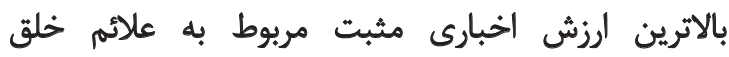

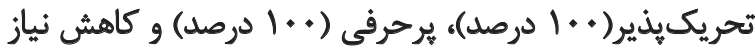

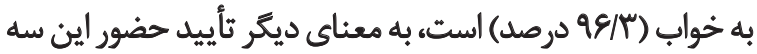

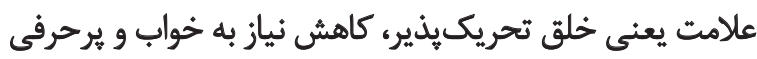

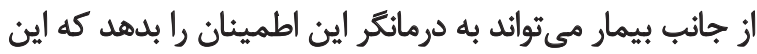

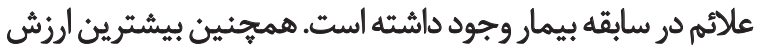

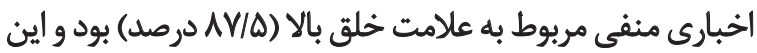

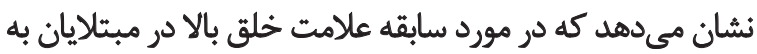
اختلال دوقطبى، به جواب منفى بيمار مان مي توان اعتماد كرد. برخى از محدوديتهاى برجسته اين مطالعه عبارتاند از

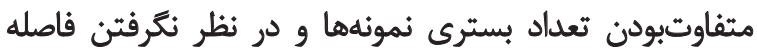

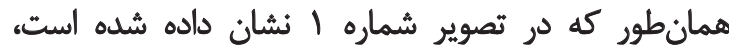

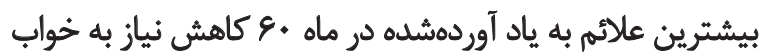

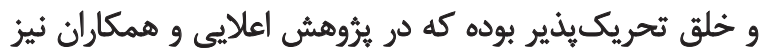

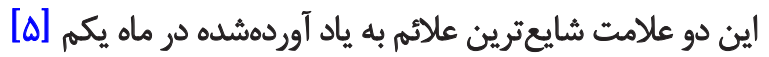

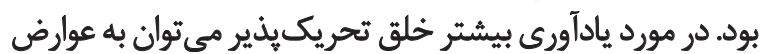

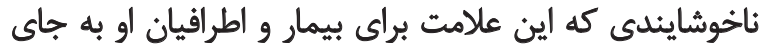

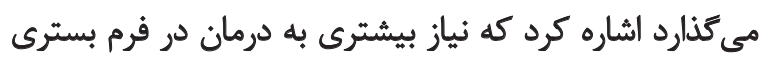

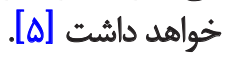

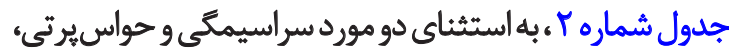

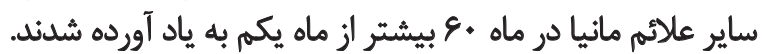

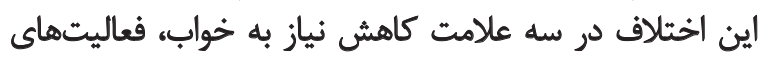

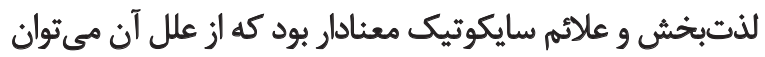

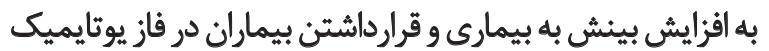

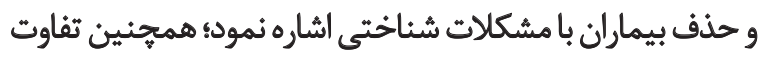

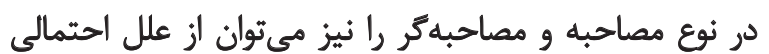

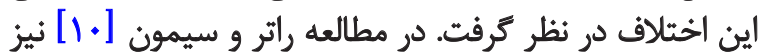

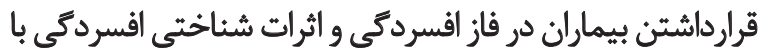

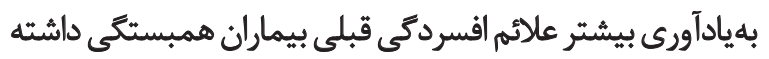

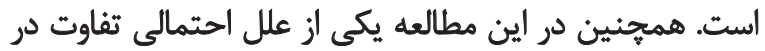

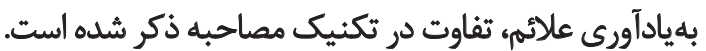

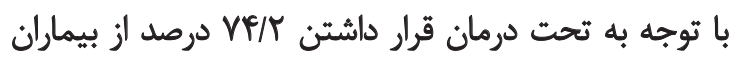

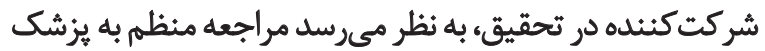

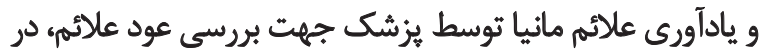

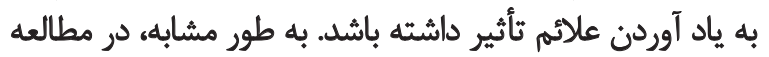

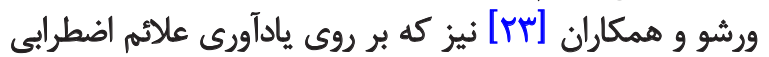

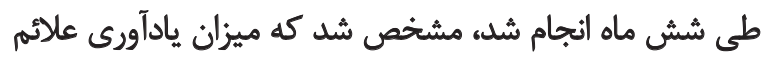

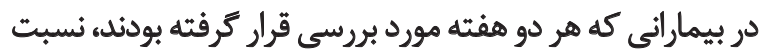

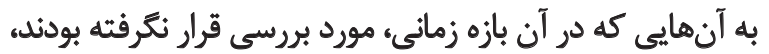

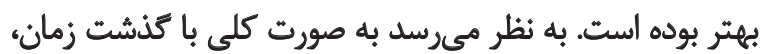

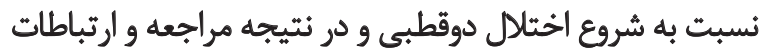

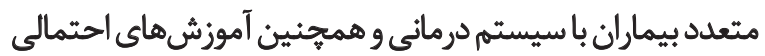

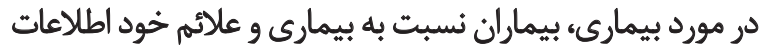

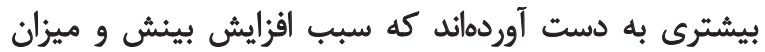

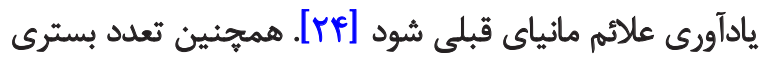

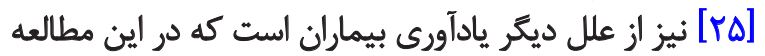
مورد بررسى قرار نتخرفت.

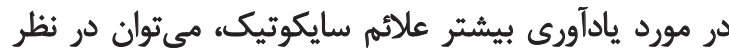

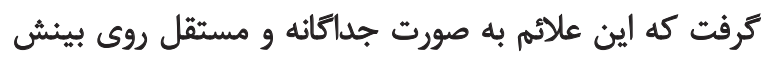

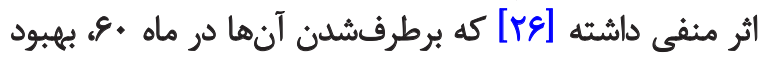
مضاعف بينش و يادآورى بيشتر علائم قبلى مانيا را به همراه آنهاه داشته است. 


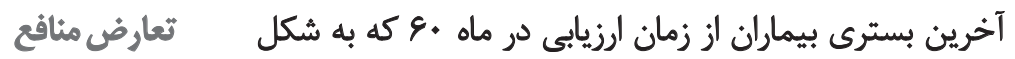
بنابر اظهار نويسندكان، اين مقاله تعارض منافع ندارد.

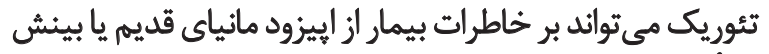

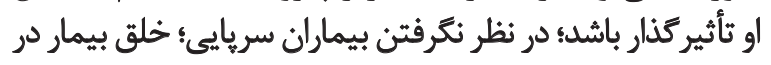

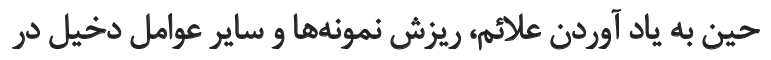

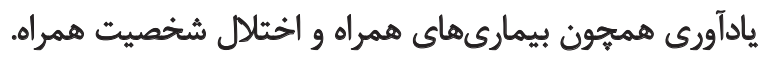

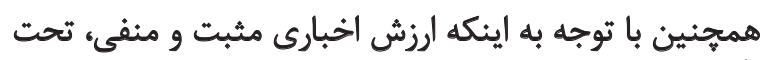

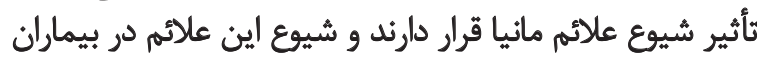

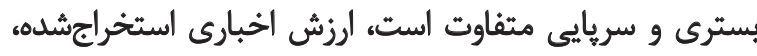
بهتنهايى ودر همه شرايط بالينى قابل اتكا نيست.

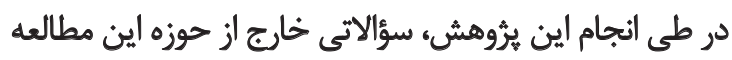

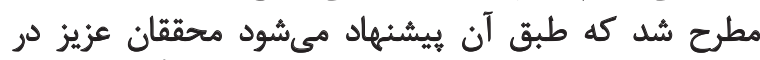

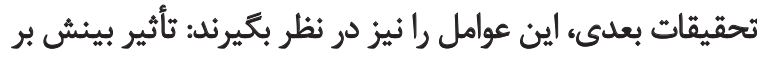

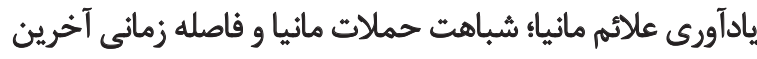

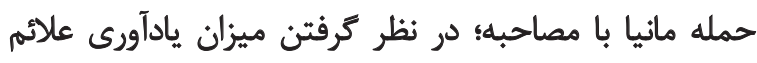

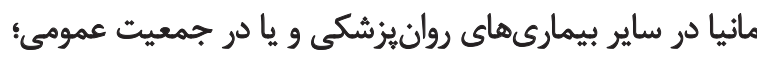

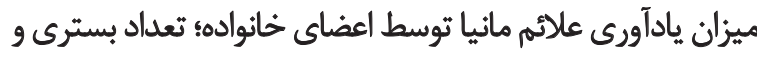

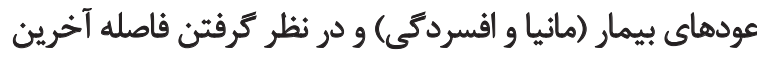

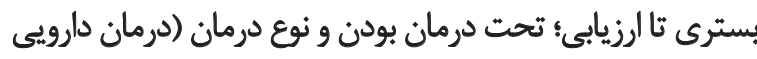

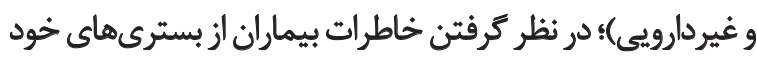

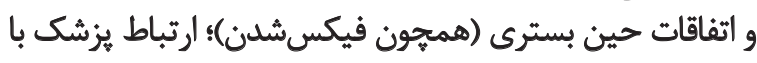

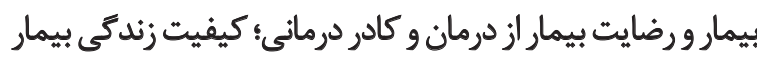

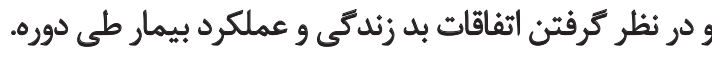

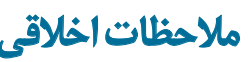

ييدوى از اصول اخلاق يُؤهش

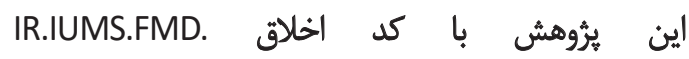

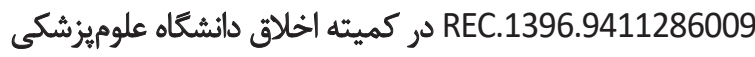
ايران تصويب شده است. همه اصول اخلافى در اين مقاله ركاله رعايت

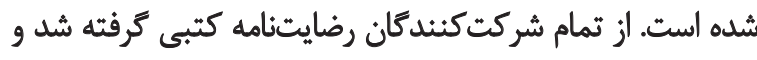

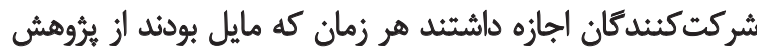

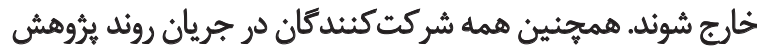
بودند. اطلاعات آنها نيز محرمانه نكه داشته شد.

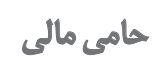

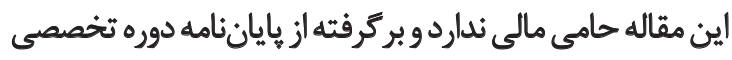

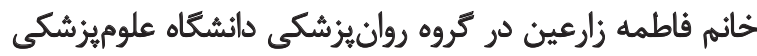

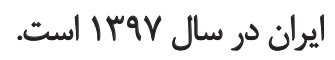

$$
\text { مشاركت نويسند مانّان }
$$

تمامى نويسندكان در آمادهازى اين مقاله به يك اندازه

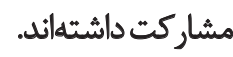




\section{References}

[1] Murray CJL, Lopez AD. Evidence-based health policy--lessons from the Global Burden of Disease Study. Science. 1996; 274(5288):740-3. [DOI:10.1126/science.274.5288.740] [PMID]

[2] Salvatore P, Tohen M, Kaur Khalsa HM, Baethge C, Tondo L, Baldessarini RJ. Longitudinal research on bipolar disorders. Epidemiology and Psychiatric Sciences. 2007; 16(2):109-17. [DOI:10.1017/S1121189X00004711] [PMID]

[3] Shabani A, Teimurinejad S, Kokar S, Ahmadzad Asl M, Shariati B, Mousavi Behbahani Z, et al. Suicide risk factors in Iranian patients with bipolar disorder: A 21-month follow-up from BDPF study. Iranian Journal of Psychiatry and Behavioral Sciences. 2013; 7(1):16-23. [PMID] [PMCID]

[4] Shirazi E, Shabani A, Hakim Shooshtari M, Ghadiri Vasfi M. [Definition of cycle and episode in rapid cycling bipolar disorder: An area of debate in diagnosis and research (Persian)]. Iranian Journal of Psychiatry \& Clinical Psychology. 2017; 23(3):254-9. [DOI:10.29252/nirp.ijpcp.23.3.254]

[5] Alaie S. [Identification of more commonly used mania acute manifestations for diagnosis of bipolar disorder in newly discharged patients, in Medical [MSc. thesis] [Persian]. Tehran: Iran University of Medical Sciences; 2014.

[6] Gharaipour M. [Neuropsychological function and its relationship with psychosocial function and clinical features in bipolar disorders (Persian)]. Psychological Studies. 2007; 3(1):71-82. https://www.sid.ir/fa/journal/ViewPaper.aspx?ID=67340

[7] Sharifi V, Asadi SM, Mohammadi MR, Amini H, Kaviani H, Semnani $Y$, et al. [Reliability and feasibility of the Persian version of the structured diagnostic interview for DSM-IV (SCID) (Persian)]. Journal of Advances in Cognitive Science. 2004; 6(1-2):1022. http://icssjournal.ir/article-1-26-en.html

[8] Ahmadvand A, Sepehrmanesh Z, Ghoreyshi F, Assarian F, Moosavi GA, Saee R, et al. [Prevalence of mental disorders in general population of Kashan City (Persian)]. Iranian Journal of Epidemiology. 2010; 6(2):16-24.

[9] Simon GE, Rutter CM. Accuracy of recall for mania symptoms using a three month timeline follow-back interview. Journal of Affective Disorders. 2008; 107(1-3):271-4. [DOI:10.1016/j. jad.2007.08.020] [PMID] [PMCID]

[10] Rutter CM, Simon G. A Bayesian method for estimating the accuracy of recalled depression. Journal of the Royal Statistical Society: Applied Statistics, Series C. 2004; 53(2):341-53. [DOI:10.1046/ j.1467-9876.2003.05211.x]

[11] Shabani A, Taheri A, Azadforouz S, Najd Abbasi Ch, Mousavi Z, Zangeneh K, et al. Bipolar Disorder Patients Follow-up (BDPF): Methods and materials. Journal of Research in Medical Sciences. 2010; 15(4):229-34. http://jrms.mui.ac.ir/index.php/jrms/article/view/4616

[12] Ebrahimi A, Taher Neshatdoost H, Mousavi SG, Asadollahi GA, Nasiri H. Controlled randomized clinical trial of spirituality integrated psychotherapy, cognitive-behavioral therapy and medication intervention on depressive symptoms and dysfunctional attitudes in patients with dysthymic disorder. Advanced Biomedical Research. 2013; 2:53. [DOI:10.4103/2277-9175.114201] [PMID] [PMCID]
[13] Zeschel E, Correll CU, Haussleiter IS, Krüger-Özgürdal S, Leopold K, Pfennig A, et al. The bipolar disorder prodrome revisited: Is there a symptomatic pattern? Journal of Affective Disorders. 2013; 151(2):551-60. [DOI:10.1016/j.jad.2013.06.043] [PMID]

[14] Vieta E. Guide to assessment scales in bipolar disorder. Tarporley: Springer Healthcare; 2010. [DOI:10.1007/978-1-907673-26-9]

[15] Barekatein M, Tavakkoli M, Molavi H, Maeroofi M, Salehi M. [Validity and reliability of Young Mania Rating Scale in Iran (Persian)]. Journal of Psychology. 2007; 11(2):150-66. https://iranjourm nals.nlai.ir/2467/article_588593.html

[16] Folstein MF, Folstein SE, McHugh PR. "Mini-mental state": A practical method for grading the cognitive state of patients for the clinician. Journal of Psychiatric Research. 1975; 12(3):189-98. [DOI:10.1016/0022-3956(75)90026-6]

[17] Anthilingam P. Validation of an instrument to measure cognitive function in patients with heart failure [PhD. disertation]. New York: University of Rochester; 2007.

[18] Lorbach ER, Webster KE, Menz HB, Wittwer JE, Merory JR. Physiological falls risk assessment in older people with Alzheimer's disease. Dementia and Geriatric Cognitive Disorders. 2007; 24(4):260-5. [DOI:10.1159/000107101] [PMID]

[19] Seyyedian M, Fallah M, Nooruzian M, Nejat S, Delavar A, Ghasemzadeh H. [Preparation and validation of the Persian version of the short test of mental status (Persian)]. Journal of Medical Council of Iran. 2007; 25(4):408-14.

[20] Abedi Gheshlaghi H, Valizadeh Kamran Kh. [Evaluation and zoning of forest fire risk using multi-criteria decision-making techniques and GIS (Persian)]. Journal of Natural Environmental Hazards. 2018; 7(15):49-66.

[21] Altman DG, Bland JM. Statistics notes: Diagnostic tests 2: Predictive values. BMJ. 1994; 309(6947):102. [DOI:10.1136/ bmj.309.6947.102] [PMID] [PMCID]

[22] Yaseri M, Yekaninejad MS, Pakpour A, Rahmani S, Rangin H Akaberi A. [Self-Learning concepts of diagnostic tests by graphical approach: sensitivity, specificity, positive predictive value and negative predictive value (Persian)]. Journal of North Khorasan University of Medical Sciences. 2012; 4(2):275-82. [DOI:10.29252/ jnkums.4.2.275]

[23] Warshaw MG, Dyck I, Allsworth J, Stout RL, Keller MB. Maintaining reliability in a long-term psychiatric study: An ongoing inter-rater reliability monitoring program using the longitudinal interval follow-up evaluation. Journal of Psychiatric Research. 2001; 35(5):297-305. [DOI:10.1016/S0022-3956(01)00030-9]

[24] Yen CF, Chen CS, Yeh ML, Ker JH, Yang SJ, Yen JY. Correlates of insight among patients with bipolar I disorder in remission. Journal of Affective Disorders. 2004; 78(1):57-60. [DOI:10.1016/ S0165-0327(02)00213-6

[25] Ghaemi SN, Hebben N, Stoll AL, Pope Jr HG. Neuropsychological aspects of lack of insight in bipolar disorder: a preliminary report. Psychiatry Research. 1996; 65(2):113-20. [DOI:10.1016/ S0165-1781(96)02956-3]

[26] Yen CF, Chen CS, Yeh ML, Yang SJ, Ke JH, Yen JY. Changes of insight in manic episodes and influencing factors. Comprehensive Psychiatry. 2003; 44(5):404-8. [DOI:10.1016/S0010440X(03)00107-X] 